\title{
A study of temporal bone fractures
}

\author{
Nitin Gupta · Saurabh Varshney · S. S. Bist · Rajat Bhatia
}

\begin{abstract}
Many young adults are now killed and injured in accidents than from another causes. More than $75 \%$ of these injuries are to the head, and the ear being the most frequently injured sensory organ of the body. Temporal bone or basilar skull fractures are extremely common in any head injury. Injuries to the temporal bone may be considered in three groups: Those affecting the external auditory meatus (extralabyranthine fractures), those largely affecting middle ear cleft (tympanolabyranthine) and those affecting the internal ear (labyrinthine fractures). Many injuries, however, involve all these structures. The sudden onset of facial paralysis, vertigo and hearing impairment after a head injury is a matter of great concern for the patients and clinicians. Presence of cerebrospinal fluid leak (CSF Otorrhoea) can be a challenge for both the neurosurgeons and otologists. We hereby present 86 patients of temporal bone fractures who presented in the departments of emergency, Neurosurgery or ENT of Himalayan Institute of Medical Sciences, Dehradun during last 10 years (1996-2006).
\end{abstract}

Keywords Temporal bone - Facial nerve palsy · CSF otorrhoea

N. Gupta - S. Varshney $(\bowtie) \cdot$ S. S. Bist • R. Bhatia

Dept. of ENT, Himalayan Institute of Medical Sciences, Swami Ram Nagar, Doiwala, Dehradun.

(U.A.) - 248 140, India

S. Varshney $(\square)$

e-mail: drsaurabh68@gmail.com

\section{Introduction}

The number of adults getting injured and killed in road traffic accidents have increased in the recent past. Head bears the brunt of majority of these road traffic accidents with more than $75 \%$ of these injuries affecting the head [6]. Ear is the most frequently injured sensory organ of the body [3]. Temporal bone or skull base fractures are extremely common in head injuries. Injuries resulting from trauma to temporal bone range from temporary and minor disorders to severe and permanent ones. These \#s are usually associated with severe neurological deficits but the auditory and vestibular systems may also be affected. Many of these patients sustain other life threatening injuries, which usually take priority in initial management. In a seriously head injured patient, it is important not to overlook the management of these non life threatening injuries in an attempt to try and improve their subsequent quality of life. Unrecognized otological complications are one such group of injuries, which, if left untreated, may lead to difficulties in rehabilitation and adversely affect the overall quality of life.

Injuries to the temporal bone may be considered in three groups [1]: those affecting the external auditory meatus (extralabyranthine fractures), those largely affecting the middle ear cleft (tympanolabyranthine fractures) and those affecting the internal ear (labyrinthine fractures). Many injuries however involve all these structures.

Fractures involving the temporal bone can be classified into longitudinal, transverse and mixed types depending on the relationship of the fracture line to the long axis of the petrous temporal bone [9]. In longitudional \#s, the \# line begins in the squamous temporal bone and extends along the roof of bony external auditory canal, tearing meatal skin and tympanic membrane and crossing the roof of middle ear [10]. It can then run anterior to labyrinthine capsule, through carotid canal to end near Foramen Spinosum. In transverse \#s, the \# line extends transversely across petrous pyramid passing through vestibule of inner ear. Myriad of presentations of these fractures consisting of Facial 
Nerve Paralysis, CSF Otorrhoea, Hearing impairment and Vertigo can be a challenge for both otolaryngologists and neurosurgeons alike.

\section{Material and methods}

This study was conducted in the Himalayan Institute of Medical Sciences,Dehradun. All these patients had presented in the ENT, Neurosurgery and emergency departments of Himalayan Institute of Medical Sciences, Dehradun and were diagnosed as cases of temporal bone fractures. Only those cases who had evidence of fractures on HRCT Temporal bone were included in the study. Total number of patients who were included in this study were 86 . The study was conducted over a period of 10 years from 1996-2006.

Age of these patients ranged between 12 years and 76 years with a mean age of 28.5 years. There were $64(76 \%)$ males and 22(24\%) females in the study. $69(80 \%)$ of these patients were victims of road traffic accidents. 33(44\%) patients gave history of being under the influence of alcohol while driving or were travelling in a vehicle driven by a drunken person. The site of impact on skull in these patients of head injuries was occipital in 34(39\%), parietal in $18(21 \%)$, temporal in $17(19 \%)$, frontal in $8(9 \%)$ and mixed in $6(7 \%)$ cases.

These patients presented with a variety of otological symptoms (Table 1).

On examination, Haemotympanum was seen in 21(24\%) patients, Tympanic Membrane perforations in 19(22\%) patients, CSF Otorrhoea in 12(14\%), Mastoid tenderness in $11(13 \%)$, Facial N. palsy (Fig. 3) in $8(9 \%)$, Nystagmus in $14(16 \%)$ and Chorda Tympani N. involvement in $2(2 \%)$ patients (Fig. 1).

HRCT was done in all these patients and showed these findings (Fig. 2).

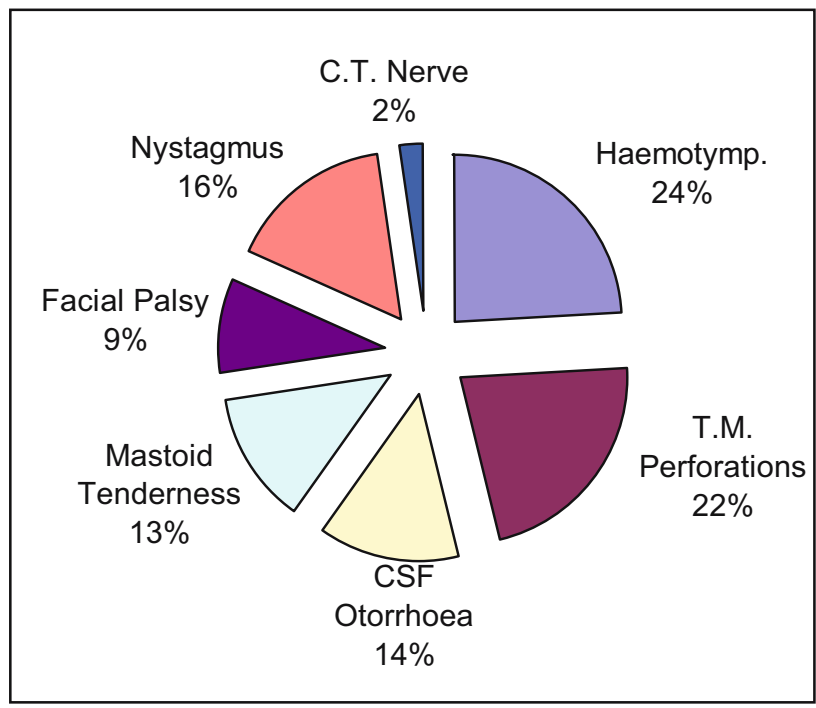

Fig. 1 Distribution of symptoms during presentation
Tuning Fork Tests were performed in these patients. Rinnes Test was negative in $43(50 \%)$ patients. Webers Test was lateralised to either of the ear in $16(17 \%)$ patients while ABC was shortened in 30(32\%) patients. Audiometric findings were suggested of sensorineural hearing loss in $28(30 \%)$, conductive loss in $28(30 \%)$ and mixed hearing loss in $6(7 \%)$ cases. High frequency SNHL was noted in $8(9 \%)$ patients while $20(23 \%)$ patients had low frequency SNHL. Profound hearing loss was found in $13(15 \%)$, severe loss in $11(12 \%)$, moderate in $2(2 \%)$ and mild SN loss in patients. Tympanometry was also done in some of these

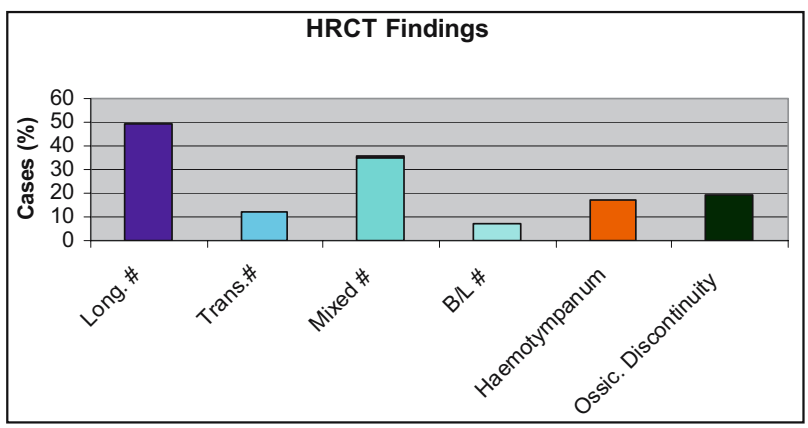

Fig. 2 Bar chat with HRCT findings

\begin{tabular}{lc}
\hline \multicolumn{2}{l}{ Table 1 Showing otological symptoms } \\
\hline Symptoms & Total cases (\%) \\
Otohaematorrhoea & $60(70 \%)$ \\
Deafness & $56(65 \%)$ \\
Vertigo & $18(21 \%)$ \\
Tinnitus & $12(14 \%)$ \\
Facial asymmetery & $06(08 \%)$ \\
\hline
\end{tabular}

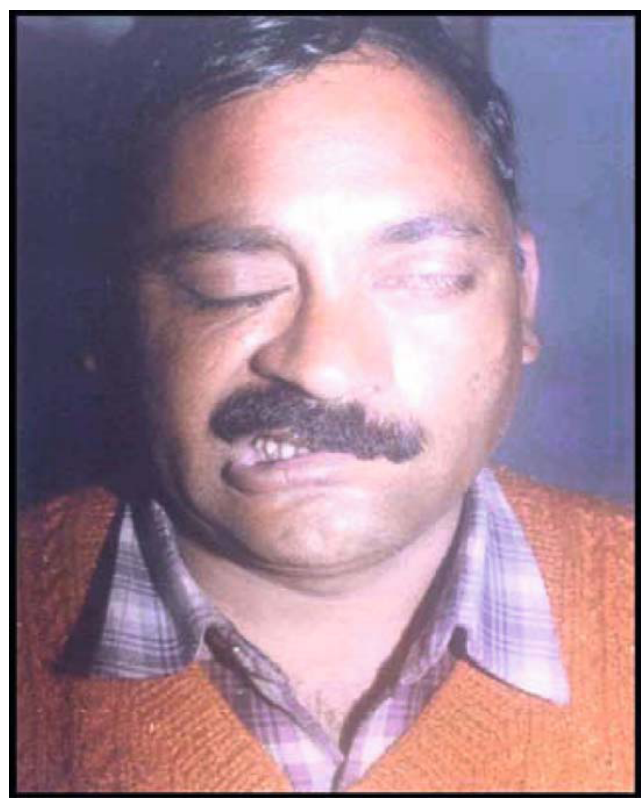

Fig. 3 Patient with Lt. Infranuclear Facial Nerve Palsy 


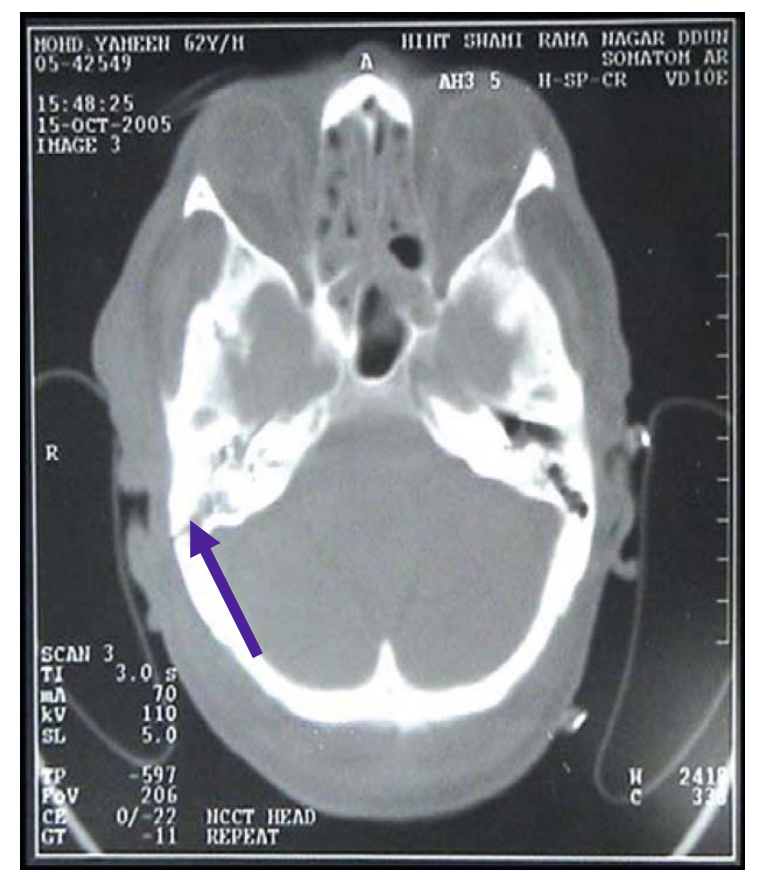

Fig. 4 HRCT Temporal bone showing oblique \# of Rt. Petrous Temporal bone

patients. It showed ' $\mathrm{B}$ ' type of curve suggestive of haemotympanum in $12(14 \%)$ cases and 'Ad' type of curve suggestive of ossicular discontinuity in 6(7\%) patiens.

Most of these patients $80(93 \%)$ were managed conservatively while $6(7 \%)$ patients required surgical intervention. A protocol of avoiding aural packs, not removing blood clots from ear canals, keeping sterile ear pads and maintaining aseptic care was followed in these patients.

Spontaneous healing of CSF Otorrhoea was seen in $10(12 \%)$ cases while $2(2 \%)$ patients required surgical intervention. Cranial approach was used to close the leak in these patients by the neurosurgeons [9].

Conductive hearing loss improved spontaneously by conservative management in $34(40 \%)$ patients. Six patients who didn't improve in 3 months time required Tympanoplasty and improved subsequently. In patients with SNHL loss, hearing aids were prescribed in $12(14 \%)$ patients while $4(5 \%)$ of SNHL loss improved spontaneously.

Facial nerve palsy was managed conservatively with steroids in $6(7 \%)$ patients while surgical exploration and decompression of the nerve was done in $2(2 \%)$ cases. Trans-mastoid approach was used to explore and decompress the nerve in these patients.

Labyranthine sedatives were prescribed for 10 days to treat vertigo. BPPV was seen in $4(5 \%)$ patients and it was self limiting.

\section{Outcome}

43(50\%) patients were followed for a period of 6 months while rest of the patients were lost to follow up. CSF
Otorrhoea did not persist in any of these patients. There was persistent Sensorineural hearing loss in $17(20 \%)$ cases while all the patients of conductive hearing loss improved spontaneously or by Tympanoplasty. Two patients persisted with Facial Nerve palsy even after 6 months follow up. Vertigo and Tinnitus continued to be present in $9(10 \%)$ patients.

\section{Discussion}

Because of high-speed travel and activities unknown to past generations, modern man faces an increasing problem of severe injuries and death due to trauma. Correctable and preventable injuries to the ear are of major concern and deserve close attention. Several important findings and observations were made in the study. Young adults are very commonly affected by these injuries. This could be due to the fact that males are more involved in outdoor activities and hence more prone to such accidents. Consumption of alcohol reduces the reflexes of the drivers and knocks down the senses and hence increases the chances of accidents $[3,8]$. Its consumption should be strictly banned while driving. Every patient of head injury should be thoroughly evaluated by otolaryngologists. Patients of ear bleed are strongly suggestive of longitudinal fractures [7]. Otological manifestations in patients of temporal \#s should be given due attention and managed properly. Otoscopic examination and audiological evaluation are necessary for proper assessment of these patients. HRCT Temporal bone (Fig. 4) is the key radiological investigation for assessing and managing these patients [9]. Transverse \#s are less common than longitudinal \#s but are associated with higher morbidity in the form of facial nerve palsy, hearing loss, vertigo [2, $3,6]$. Otohaematorrhoea and hearing loss are the common presentations of these patients. Conductive hearing loss is caused by tympanic membrane perforation, haemotympanum or ossicular disruption [5]. These patients of conductive hearing loss generally improve spontaneously but Tympanoplasty should be considered in those patients in whom the hearing loss persists for more than 3 months. Sensorineural hearing loss is a major handicap for these patients. Hearing Aids should be prescribed for such patients. CSF Otorrhoea generally heals spontaneously in majority of patients in 2 weeks time. Neurosurgical help should be sought if leak persist for a longer duration. Facial nerve palsy should be managed conservatively with the help of steroids but complete and immediate onset palsy strongly suggests facial nerve transection [8,9]. Exploration of Facial nerve should not be delayed in these patients. Rehabilitation of these patients require proper management of SNHL, tinnitus and vertigo and these otological symptoms should not take a backseat.

\section{References}

1. Pollanen MS, Deck JHN, Blenkinsop B, Farkas EM (1992) Fracture of temporal bone with exsanguination: pathology and mechanism. Can J Neurol Sci 19:196-200 
2. Griffiths MV (1979) The incidence of auditory and vestibular concussion following minor head injury. J Laryngol Otol 93: 253-226

3. Tos M (1971) Prognosis of hearing loss in temporal bone fracture. J Laryngol Otol 85(1)147-1, 159

4. Schubiger O, Valavanis A, Stuckmann G, Antonucci F (1986) Temporal bone fractures and their complications. Neuroradiology 28:93-99

5. Kochhar LK, Deka RC, Kacker SK, Raman EV (1990) Hearing loss after head injury. Ear, Nose and Throat Journal 69: $537-542$
6. Coker N, Kendall K, Jenkins H, et al (1987) Traumatic intratemporal facial nerve injury: management rationale for preservation of function. Otolaryngol Head Neck Surg 97: 262

7. Jack VD,McGee M. Otologic Trauma. Otolaryngology 3rd edition (2):1137-1160

8. Kamere BD Temporal bone trauma. Operative Otolaryngology-Head\& Neck Surgery. 1st edition (2):1537-1548

9. Lancaster JL, Alderson DJ, Curley JW Otological complications following skull base fractures. J Coll Surg 44(2): $87-90$ 